\title{
Mitteilungen der Mitteldeutschen Gesellschaft für Gastroenterologie
}

\section{Gastroenterologe 2020 · 15:69}

https://doi.org/10.1007/s11377-019-00412-y

(c) Springer Medizin Verlag GmbH, ein Teil von Springer Nature 2020

Mitteldeutsche Gesellschaft für Gastroenterologie Hessen - Sachsen - Sachsen-Anhalt - Thüringen
Mitteldeutsche Gesellschaft für Gastroenterologie e.V. Hessen · Thüringen · Sachsen · Sachsen-Anhalt

\section{Schriftführer}

Prof. Dr. med. Joachim Glaser

Vitalisklinik Bad Hersfeld GmbH

Am Weinberg 3

36251 Bad Hersfeld

\section{Redaktion}

J. Hampe, Dresden (1. Vorsitzender)

H. J. Glaser, Bad Hersfeld (Schriftführer)

\section{MGG 2020}

\section{5.- 6. Juni 2020, Dresden}

\section{Liebe Kolleginnen und Kollegen,}

wir freuen uns sehr, Sie zur dritten gemeinsamen Jahrestagung der Mitteldeutschen Gesellschaft für Gastroenterologie (MGG) und der Mitteldeutschen Chirurgenvereinigung (MDCV) in Dresden zu begrüßen. Nach den erfolgreichen gemeinsamen Kongressen 2018 in Leipzig und 2019 in Halle wollen wir das interdisziplinäre Profil des wissenschaftlichen Programms weiter stärken. Gemäß unserem Kongressmotto "Innovationen für die Praxis“ haben wir zeitgemäße Themenschwerpunkte in den Bereichen innovativer operativer und gastroenterologischer Therapien, der Onkologie, bei seltenen und bei entzündlichen Erkrankungen des Gastrointestinaltrakts, sowie dem immer relevanteren metabolischen Syndrom gesetzt.

Besonders wichtig ist es uns, die Interessen der jungen Chirurgen und jungen Gastroenterologen in unserem Programm abzubilden. Wir freuen uns auf Ihre zahlreiche Teilnahme in den dezidierten Sitzungen und praktischen Workshops unter anderem in den Bereichen Robotik, Naht und Darmsonographie. Herausragende Leistungen würdigen wir traditionell durch die im Rahmen des Gesellschaftsabends verliehenen Posterpreise.

Wir finden, Viszeralmedizin ist Teamwork. Daher steht die Einbeziehung der Pflege mit dem bewährten Pflegesymposium wieder auf dem Programm und soll in interdisziplinären Workshops weiter gestärkt werden.

Mit dem Internationalen Congress Center Dresden steht uns ein sehr attraktiver Veranstaltungsort zur Verfügung. Neben hervorragenden Ausstellungsmöglichkeiten und modernster Ausstattung bietet die zentrale Lage die Gelegenheit die historische Altstadt zu erkunden. Eine weitere Perspektive auf das schöne Elbflorenz wird sich uns beim Gesellschaftsabend bieten, den wir bei einer abendlichen

Alle Informationen zu Kongressprogramm, Autoren, Moderatoren finden Sie unter https://www.mdcv-mgg.de. Informationen auf Ihr Smartphone oder Tablet: Bitte scannen Sie den QR-Code für Ihren Zugriff auf das Mobil-Geräte-freundliche OnlineProgramm

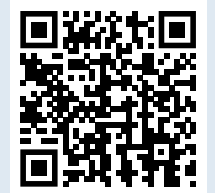

Dampferfahrt mit Gelegenheit zum Austausch in ungezwungener Atmosphäre verbringen werden.

Wir freuen uns auf einen spannenden Kongress mit Ihnen vom 5.-6. Juni 2020 in Dresden!

Mit herzlichen Grüßen Ihre
Prof. Dr. med. Jochen Hampe Kongresspräsident 2020 der Mitteldeutschen Gesellschaft für Gastroenterologie

Prof. Dr. med. Jürgen Weitz Kongresspräsident 2020 der Mitteldeutschen Chirurgenvereinigung
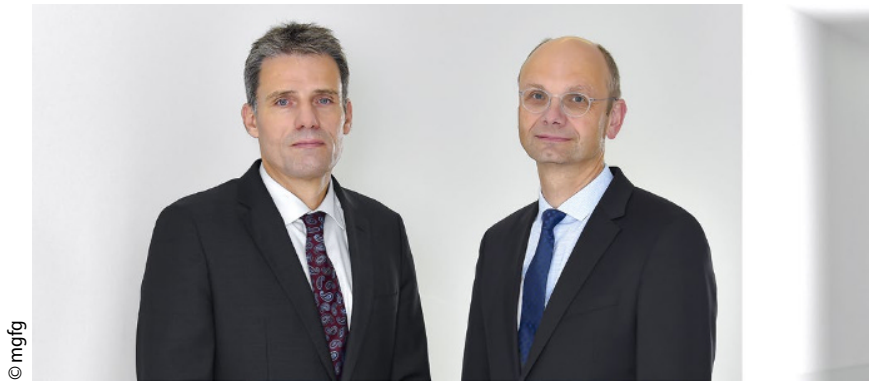